\title{
NUCLEI FAMILIARI TRA PERSONE SAME-SEX: LESPERIENZA GIURIDICA ITALIANA A CONFRONTO CON QUELLA SPAGNOLA QUADRO INTRODUTTIVO DELLA PROBLEMATICA
}

\author{
Elisa Dolzini \\ Universitá di Bergamo
}

\section{RESUMEN}

Este trabajo analiza, comparativamente, las diferencias temporales y conceptuales que han caracterizado la respuesta legislativa de Espańa e Italia a la regulación de uniones entre personas del mismo sexo. La normativa y la jurisprudencia han evolucionado de forma distinta y, pese a las afinidades socioculturales y jurídicas existentes entre ambos Estados, sigue habiendo diferencias significativas que se extienden también a la regulación de las adopciones por parejas del mismo sexo.

Palabras Clave: matrimonio homosexual, adopción.

\section{SAME-SEX UNIONS IN SPAIN AND ITALY}

\section{Abstract}

This paper analyzes, comparatively, the temporal and conceptual differences that have characterized the legislative response of Spain and Italy to the regulation of unions between people of the same sex. Regulations and jurisprudence have evolved differently and, despite the existing socio-cultural and legal affinities between both States, there are still significant differences that also extend to the regulation of adoptions by same-sex couples.

KeYwords: Same sex marriage, adoption. 


\section{QUADRO INTRODUTTIVO DELLA PROBLEMATICA}

L'Italia, paese di eccellente e prestigiosa tradizione giuridica, da cui spesso la dottrina spagnola ha attinto per l'evoluzione dei propri orientamenti, si trova, con riferimento alle unioni omoaffettive, fanalino di coda ${ }^{1}$ nel panorama giuridico europeo $^{2}$.

La Spagna, paese di radici socio-culturali affini e molto simili a quelle italiane, e nel quale è forte, come in Italia, l'influenza della tradizione cattolica nelle scelte politico-legislative, aveva, già a partire dal 2005, legiferato circa dette unioni, ampliando l'istituto del matrimonio già costituzionalmente e codicisticamente disciplinato, in modo da ricomprendere nello stesso la possibilità per persone dello stesso genere di contrarre tra loro matrimonio (estendendo così anche a dette coppie la possibilità di adozione filiale).

Nonostante l'Italia non sia certo da considerare un Paese giuridicamente arretrato, ovvero insensibile alle questioni etiche e bioetiche (si pensi al recente orientamento giurisprudenziale nazionale a proposito della fecondazione eterologa e al recente decreto di aggiornamento delle linee guida della legge n. 40/2004 ${ }^{3}$ ), per ragioni che in questa sede non rileva analizzare specificamente -si evidenzi, ad ogni modo, il quadro di equilibri politici precari che hanno caratterizzato la più recente storia nazionale-, solo nell'anno appena conclusosi ${ }^{4}$ il Parlamento italiano (con un

${ }^{1}$ L'Italia, dopo un lungo periodo di inerzia legislativa, è l'ultimo Paese dell'Unione Europea ad aver legiferato circa le unioni omoaffettive: dopo l'intervento legislativo italiano, il vuoto permane oggi solamente in Bulgaria, Lettonia, Lituania e Romania; altresì in Slovacchia e Polonia, dove però giace in Parlamento un disegno di legge.

2 Per un approfondimento in ottica non solo nazionale, ma comparatistica si cfr.: «Famiglia e matrimonio", vol. I, in Trattato di diritto di famiglia, diretto da Paolo Zatti, Seconda ed., Giuffrè, Milano, 2011, p. 793 e ss.

${ }^{3}$ La legge n. 40/2004, rubricata "Norme in materia di procreazione medicalmente assistita», è stata modificata prima dalla sentenza della Corte Costituzionale n. 151/2009 -che ha eliminato il numero massimo di embrioni trasferibili in un unico impianto-e poi dalla sentenza 162/2014 -che ha eliminato il divieto di fecondazione eterologa, rendendo pertanto così legale e fruibile detta pratica medica, pratica ad oggi ancora non consentita dalla legislazione spagnola.

${ }^{4}$ Per comprendere l'ingente ritardo dell'intervento legislativo nazionale (pacificamente inficiato dalla posizione conservatrice delle correnti politiche maggiormente numerose, storiche e con rilevante peso politico) rispetto al quadro europeo in cui si inserisce la tradizione giuridica italiana, è significativo riflettere sul fatto che, già nel 1989, in Danimarca (Paese avanguardista e, per così dire, "pioniere» nelle questioni bioetiche, in generale, e, in particolare, nella tutela dei diritti delle coppie same-sex) era possibile registrare le Unioni omoaffettive e, nel 1999, la legge n. 360 introduceva nella legislazione danese la stepchild adoption per il partner registrato che intendesse adottare il figlio minore del proprio partner, salvo che questi fosse stato già adottato in un altro Paese-Merin Y. Equality for same-sex Couples: the legal Recognition of Gay Partnership in Europe and the United States, University of Chicago Press, Chicago, 2002, p. 61 e ss.-; in Italia, nello stesso anno (1999), uno dei più diffusi manuali di diritto di famiglia con riferimento alle unioni omoaffettive così si esprimeva: "un simile fenomeno non pare possa essere reputato contra legem», invero, non pare potersi procedere oltre un «atteggiamento di indifferenza dell'ordinamento»- Quadri E., Famiglia e Ordinamento civile, seconda ed., Giappichelli, Torino, 1999, p. 32 e ss. 
iter tormentato conclusosi con approvazione della legge sottoposta all'apposizione della fiducia da parte del Governo in entrambe le Camere) ha disciplinato, nel maggio scorso, le unioni omoaffettive, con la legge n. 76/2016 rubricata: "Regolamentazione delle unioni civili tra persone dello stesso sesso e disciplina delle convivenze». Undici anni di ritardo, quindi, rispetto all'intervento legislativo spagnolo, decisamente più rivoluzionario (rispetto a quello italiano) che, rompendo con la tradizione dottrinale e giurisprudenziale, non decide per una legge ad hoc che disciplini le unioni tra persone dello stesso sesso, bensì, con la legge (ley) n. 13/2005, estende alle stesse la possibilità di contrarre matrimonio: audace decisione politica con note e decisive ripercussioni sociali -negli anni successivi all'introduzione di detta legge- circa la considerazione delle unioni omoaffettive (ripercussioni sociali che a loro volta influenzano la considerazione giuridico-politica dell' istituto all'interno della società; quelli ora citati sono, infatti, aspetti correlati tra loro che si influenzano a vicenda dando così vita, a seconda delle circostanze, a circoli viziosi/virtuosi).

Tornando all'Italia, ai plurimi moniti della giurisprudenza di legittimità, della Corte Costituzionale e della Corte Edu, che insistentemente sollecitavano il Parlamento italiano al fine di colmare questo vuoto legislativo ${ }^{5}-\mathrm{e}$ culminati in una condanna per l'Italia con la sentenza Oliari e al. C. Italia ${ }^{6}-$, il Parlamento

5 Non staremo, per motivi di pertinenzialità, ad analizzare né la problematica della registrazione dei matrimoni same-sex contratti all'estero e dell'annullamento, nel 2015, -su ordine del Ministro dell'Interno italiano Angelino Alfano- da parte della prefettura di dette registrazioni, effettuate dai sindaci di alcune città italiane tra cui Roma, contenzioso risolto con sentenza del CDS n. 5029/2015 -Per una miglior comprensione della problematica si cfr. sul punto: Sánchez Jordán, «El matrimonio entre personas del mismo sexo celebrado en el extranjero y su reconocimiento en Italia: un debate abierto", Revista Doctrinal Aranzadi Civil-Mercantil 9/2015, p. 59 e ss.-; né si analizzerà l'eventuale possibilità (negata) per i succitati matrimoni di essere riconosciuti con sentenza in Italia. Si evidenzia sul punto la sentenza della Corte di Cassazione n. 4184/12, che riconosce l'esistenza (pur improduttiva di effetti giuridici per l'ordinamento italiano) del matrimonio omosessuale contratto all'estero, richiamando nella propria decisione la sentenza Schalk e Kopf c. Austria del 2010 (ricorso n. 30141/04). La Corte EDU qui rigetta la possibilità che il riferimento testuale a "uomini e donne» contenuto nell'art. 12 CEDU possa essere superato con un'interpretazione evolutiva della norma, pur evidenziano come l'art. 9 della Carta dei diritti fondamentali dell'Unione Europea, non menzioni la differenza sessuale tra i coniugi come condizione necessaria per contrarre matrimonio. I giudici affermano (come nella successiva sentenza citata Oliari c. Italia) che spetta alla legislazione nazionale disporre nel senso dell'introduzione del matrimonio omosessuale ovvero altra forma di tutela dell'unione omosessuale, sottolineando come dall'interpretazione della CEDU non possa derivare, allo stato attuale, alcuna censura nei riguardi dello stato che decidesse di non procedere in questo senso. Pur non riconoscendo quindi, una violazione dell'art. 14 CEDU nella non previsione da parte della legislazione statale della possibilità di contrarre matrimonio per la coppie omosessuali, evidenzia come lo Stato debba prevedere un istituto a tutela delle unioni omoaffettive, affermando che le unioni omosessuali si trovano in una situazione simile a quella delle coppie eterosessuali quanto all'esigenza di riconoscimento e protezione giuridica della relazione.

${ }^{6}$ CEDU 21 luglio 2015, ric. 18766/11 e 36030/11: condanna, assunta a maggioranza, dell'Italia per inadempimento di obbligazione consistente nell'introduzione nella legislazione nazionale di una disciplina normativa delle unioni same-sex; Corte Edu che lascia al libero apprezzamento dei singoli Stati la scelta del tipo di intervento legislativo (apertura ed estensione dell'istituto del matrimonio tra persone dello stesso genere ovvero legislazione ad hoc circa l'unione omoaffettiva), ma 
nazionale ha risposto, dopo lungo ed acceso dibattito politico-sociale (dibattito sorto, socialmente, molti anni or sono, ma giunto all'agenda politica con evidente e già evidenziato ritardo), con una legge più che mai frutto del compromesso tra le diverse forze politiche, che disciplina le unioni civili (omosessuali) e le convivenze di fatto (eterosessuali ovvero omosessuali). Rileva sottolineare, ad abundantiam, che la natura compromissoria del risultato legislativo, si può facilmente evincere non solo dai contenuti della legge 76/2016, ma anche dal fatto che, ai fini dell'approvazione di detta legge, dalla stessa -in ultima battuta- sia stato stralciato quello che nel disegno di legge (d.d.l. Cirinnà) fu l'art. 5. Esso si riferiva alla possibilità, per l'unito civilmente, di adottare, previo parere giudiziario, il figlio naturale ovvero adottivo del partner a lui/lei unito civilmente (stepchild adoption) ${ }^{7}-$ si specifica, ad ogni modo, che nella legge approvata è rimasto, all'art. 1 c. 20 , ultimo periodo ${ }^{8}$, un rimando alla legge sull'adozione n. 184/83, che si andrà ad analizzare in seguito-.

Rileva puntualizzare, per completezza di informazione, come dalla legge sia stato espunto altresì il dovere di fedeltà reciproco tra i partner (il motivo dello stralcio è alquanto nebuloso).

Resta quindi precluso, alle persone che intrattengono una relazione omoaffettiva e che vogliono dare valenza civile alla propria unione, l'istituto del matrimonio, così come costituzionalmente disciplinato; d'altro canto, la legge in esame equipara espressamente la locuzione "unito civilmente» alla parola "coniuge» tutte le volte che essa compare nel codice civile; equivalenza giuridica che non sussiste, però, con riferimento alle norme che disciplinano l'adozione, disciplina da cui gli uniti civilmente rimangono esclusi. Come precedentemente accennato, infatti, la disciplina dell'adozione, per le unioni, non solo non è stata prevista, ma l'unico riferimento diretto all'istituto dell'adozione contenuto nella legge (attraverso l'istituto della "stepchild adoption») è stato stralciato; toccherà quindi, verosimilmente -come già è avvenuto fino all'avvento legislativo-, alla giurisprudenza farsi carico di questa delicata problematica, con un'ulteriore complicazione da affrontare: ora i giudici dovranno decidere non solo nel silenzio della legge, ma anche con la consapevolezza che il potere legislativo, non solo non ha disciplinato la materia dell'adozione nell'ambito

che condanna, come successo con l'Italia, l'inerzia parlamentare e il vuoto legislativo. Si evidenzia, infatti, come il già citato art. 9 della dei diritti fondamentali dell'Unione Europea garantisca il diritto a sposarsi e a costituire una famiglia «secondo le leggi nazionali che ne disciplinano l'esercizio».

7 Detto articolo, modificando la legge n. 184/83 sulle adozioni, avrebbe consentito all'unito civilmente di adottare il figlio naturale del partner; l'espunzione di detto articolo dal testo di legge è stato il fulcro del discrimine tra approvazione/non approvazione della legge Cirinnà, tanto che si è resa necessaria la presentazione in Senato di un maxiemendamento (causa della peculiare struttura legislativa -un articolo, 69 commi-) unitamente, come già precedentemente specificato, all'apposizione della fiducia (doppia) all'approvazione della legge da parte del Governo.

${ }^{8}$ Art. 1, c. 20, ultimo periodo, 1.n. 76/2016: «Resta fermo quanto previsto e consentito in materia di adozione dalle norme vigenti». 
dell'unione civile, ma ha anche stralciato la stepchild adoption dal disegno di legge (d.d.l. Cirinnà) al fine dell'approvazione dello stesso'.

Nonostante questa possa essere definita come la più grande riforma del diritto di famiglia italiano dopo il 1975, essa consta di un passaggio giuridicamente evolutivo solo eventualmente prodromico al raggiungimento di un quadro legislativo come quello spagnolo con riferimento alle unioni same-sex; pur partendo -entrambi gli Stati- da un dato costituzionale molto simile (anzi, da un punto di vista meramente letterale quello spagnolo pare essere dotato di cornice semantica ancora più «stringente» di quella ascrivibile al dettame italiano), il Parlamento spagnolo ha scelto di ampliare l'istituto del matrimonio offrendo anche alle coppie dello stesso sesso la possibilità di contrarre il suddetto, estendendo, pertanto, alle medesime, i diritti -e lo status sociale- in esso contenuto.

Vediamo quindi come, due Paesi di tradizioni storico-socio-giuridico-culturali simili e affini abbiano scelto, per il riconoscimento delle unioni same-sex, rispettivamente -nella gamma delle soluzioni possibili offerte dalla Corte Edusoluzioni antipodiche.

La Corte infatti, come precedentemente specificato in nota, pur lasciando al libero apprezzamento dei singoli Stati le modalità di risoluzione del vuoto legislativo in esame, condanna l'inerzia legislativa e chiede che gli Stati riconoscano le unioni omoaffettive.

L'Italia, con l'intervento legislativo del maggio scorso, riconosce una tutela a dette unioni, se non minima, quantomeno inferiore rispetto a quella riconosciuta alle stesse dal Parlamento spagnolo, che riconosce alle relazioni omoaffettive il massimo grado di tutela possibile, aprendo alle stesse l'istituto del matrimonio, ex art. 32 della Costituciòn española.

\section{IL DIRITTO AL RICONOSCIMENTO GIURIDICO \\ DELLE RELAZIONI OMOAFFETTIVE NELLA GIURISPRUDENZA COSTITUZIONALE: I DUE PAESI A CONFRONTO}

\section{La Sentenza del Tribunal Constitucional 198/2012}

Il Plenum del Tribunal Constitucional de España ${ }^{10}$ ha respinto (dopo sette anni, a riprova, in Spagna come in Italia, della criticità socio-politica della tematica in esame, ma con prese di posizioni di indubbio coraggio), con la sentenza $\mathrm{n}$. 198/2012, il recurso de inconstitucionalidad (ricorso in via diretta) ${ }^{11}$ n. 6864-2005,

9 Circa le problematiche derivanti da ingerenza e commistione di poteri, si cfr.: A. Cerri, «Poteri (divisione dei)», voce in Enc. Giur., Istituto Poligrafico Zecca di Stato, Roma, 1990, 1 e ss. (ad vocem)

${ }^{10}$ D'ora in avanti TC.

${ }^{11}$ Le modalità di ricorso al Tribunal Constitucional differiscono da quelle con cui ricorrere alla Corte Costituzionale; l'art. 32 della LOTC (Ley Orgànica sobre el Tribunal Constitucional) 
proposto dalla minoranza parlamentare avverso la legge n. 13/2005, che ha esteso la possibilità di contrarre matrimonio alle coppie omosessuali , modificando il Código Civil, e non già -come dagli stessi sostenuto- l'art. n. $32^{12}$ della Constitución Española (detti parlamentari lamentano nel ricorso l'interpretazione creativa -anche in Spagna, come in Italia, vietata- della stessa).

Senza ora analizzare tutti i motivi di ricorso, rileva sottolineare tra questi quello in cui i ricorrenti si dogliano, con l'introduzione della succitata Ley -e con la stessa del nuovo art. 44, c. $2,{ }^{13}$ del Código Civil- della violazione -in termini di interpretazione creativa (ivi sopra citata)- dell'art. 32, c. 1, della Costitución Española, che fa espresso riferimento all'uomo e alla donna, deducendo da tale espressione una riserva costituzionale del matrimonio a favore delle sole coppie eterosessuali, pertanto non già superabile con riserva di legge ordinaria, bensì solo con una riforma costituzionale.

A sostegno di questo orientamento, affermano altresì come la decisione del legislatore in questa sede impugnata non sia conforme al concetto di garantía istituciona $1^{14}$, che pur non assicurando un contenuto fissato e immodificabile di un determinato istituto, proprio in virtù della centralità sociale dello stesso -istituto che, pertanto, con la stessa società cresce e si evolve- deve vedere garantito e conservato il proprio nucleo identificativo, da un punto di vista ontologico (proprio per l'immagine, il «vestito", che nella coscienza sociale di ogni tempo e luogo gli è stato «cucito" addosso).

Orbene, il TC, con un dispositivo ricco di riferimenti sovranazionali e di diritto comparato, in risposta al motivo di impugnazione sopra esposto afferma, primariamente, che l'art. $32 \mathrm{CE}$ ha un duplice contenuto: esso si riferisce al matrimonio quale garanzia costituzionale, e al matrimonio come diritto costituzionale. Specifica, altresì, che, se è vero che il concetto di garantía costitucional richiede che all'istituto coperto da detta garanzia sia conferita una tutela tale da non inficiarne l'immagine che dello stesso viene riflessa, è opportuno analizzare se la legge impugnata abbia

consente alla minoranza parlamentare - che ha votato avverso l'approvazione di una legge-di ricorrere in via diretta avverso la stessa entro il termine perentorio di tre mesi dalla sua pubblicazione. Per un approfondimento sul sistema di giustizia costituzionale spagnolo si cfr.: R. Romboli, R. Tarchi "Giustizia Costituzionale in Spagna» in J.Luther, R. Romboli, R. Tarchi, (a cura di), Esperienze di giustizia costituzionale, II, Giappichelli, Torino, 2000, p. 323 e ss.

12 Art.32 della Costitución Española: «1. El hombre y la mujer tienen derecho a contraer matrimonio con plena igualdad jurídica. 2. La ley regulará las formas de matrimonio, la edad y capacidad para contraerlo, los derechos y deberes de los cónyuges, las causas de separación y disolución y sus efectos".

${ }^{13}$ Art. 44, CC: «El hombre y la mujer tienen derecho a contraer matrimonio conforme a las disposiciones de este Código. El matrimonio tendrá los mismos requisitos y efectos cuando ambos contrayentes sean del mismo o de diferente sexo".

${ }^{14}$ La teoria istituzionale, ripresa da Carl Schmitt, viene per la prima volta introdotta nella dottrina giuridica spagnola nell'ambito del dibattito sulle autonomie locali, e ha come obiettivo la delimitazione delle istituzioni giuridiche, al fine di stabilire il senso dei concetti utilizzati dal diritto positivo. Si cfr. sul punto: R. Alexy, Teoria de los derechos fundamentales, Centro de Estudios Costitucionales, Madrid, 1997, pp. 544-546. 
«stravolto» la proiezione dell' istituto del matrimonio nell'ordinamento giuridico e, quindi, se il significato del matrimonio in sede legale sia conforme al significato del medesimo in sede costituzionale, alla luce della Ley n. 13/2005.

Riferendosi anche all'interpretazione evolutiva fornita dalla Corte Edu dell'art. $12^{15}$ della Convenzione, il TC spiega come la nuova interpretazione non sia creativa, bensì evolutiva. Infatti, la stessa non è in contrapposizione con il passato, ma, confermando le proprie radici tradizionali, si evolve ampliandosi. Infatti, sostiene il TC, a seguito di un evidente evoluzione socio-culturale, non solo nazionale, bensì di respiro più ampio, lungi dal venire meno della garantía istitucional, il matrimonio è oggi «una comunità di affetto che genera un vincolo, una società di reciproco aiuto fra due persone che occupano una medesima posizione giuridica all'interno di detta istituzione e che volontariamente decidono di unirsi in un progetto di vita familiare comune».

\section{L'EVOLUZIONE GIURISPRUDENZIALE COSTITUZIONALE ITALIANA È, INVECE, DI TUTT'ALTRO TENORE}

Se il Tribunal Costitucional è intervenuto in seguito ad atto legislativo, la Corte Costituzionale è intervenuta in forza di un vuoto legislativo, auspicando e chiedendo un intervento parlamentare, pur ribadendo, con la sentenza 138/2010 prima, e con la sentenza 170/2014 poi, che il matrimonio è istituto riservato alle sole coppie eterosessuali.

Le due sentenze definiscono e consolidano, allo stesso tempo, lo status costituzionale della famiglia fondata sul matrimonio tra un uomo e una donna, e lo status costituzionale dell'unione omosessuale, quali istituti di pari rango costituzionale ma ontologicamente diversi.

Pur specificando come un'estensione dell'istituto del matrimonio alle coppie omosessuali risulterebbe contra legem, in quanto l'art. 29 Cost. ${ }^{16}$ riserva l'istituto del matrimonio alla famiglia quale comunità formata da un uomo e una donna, così come consolidatosi nella tradizione (e un'interpretazione diversa, nel senso prima espresso, sarebbe creativa, e pertanto vietata), le due sentenze chiamano (più flebilmente nel 2010, a gran voce nel 2014) l'intervento del legislatore ${ }^{17}$, quale

15 Art. 12: «A partire dall'età minima per contrarre matrimonio, l'uomo e la donna hanno il diritto di sposarsi e fondare una famiglia secondo le leggi nazionali che regolano l'esercizio di tale diritto». Nonostante il dettame letterale, la Corte, attraverso un'interpretazione evolutiva, si è espressa, più volte, sulla conformità a Convenzione del matrimonio omosessuale.

${ }^{16}$ Contra: M. Segni, «Unioni civili: non tiriamo in ballo la Costituzione», in Nuova Giur. Civ. Comm., 2015, CEDAM, II, 707 e segg., spec. 714-715.

17 Dette sentenze sono state molto rilevanti (insieme alla sentenza della Corte Edu Oliari c. Italia di cui si è precedentemente parlato) al fine di sanzionare l'inerzia del legislatore e sollecitarne l'intervento; basti pensare che il comma 1 della legge n. 76/2016 è la pedissequa ripetizione di uno stralcio della sentenza 138/2010. 
organo deputato a definire la cornice giuridica entro cui inquadrare le relazioni omoaffettive, specificando come le differenze tra i due regimi (matrimonio e unione civile) debbano superare il vaglio dell'art. 3 Cost. (criterio della ragionevolezza) ${ }^{18}$. Alle unioni omoaffettive si devono pertanto estendere tutte quelle tutele giuridiche sancite per l'istituto del matrimonio con riferimento agli ambiti in cui i due tipi di relazione risultano omogenee ${ }^{19}$, per una piena attuazione dei diritti fondamentali costituzionalmente previsti a tutela delle relazioni omoaffettive.

\section{IL CONTENUTO LEGISLATIVO DELLA LEGGE CIRINNÀ}

Se pare inutile in questa sede indugiare sull'esplicitazione del contenuto dell' istituto del matrimonio spagnolo (che si può dare per pacifico, in quanto pressoché sostanzialmente assimilabile a quello dell'istituto del matrimonio italiano -fatto salvo quanto previsto dal Código Civil spagnolo e dal Codice Civile italiano relativamente al nome familiare e alle modalità di scioglimento del matrimonio-), risulta, invece, interessante comprendere come si sviluppi -dal punto di vista contenutistico- la legge del Parlamento italiano n. 76/2016, disciplinando la stessa ad hoc le unioni omoaffettive ${ }^{20}$.

\section{CostituZione Dell'Unione Civile E PERfEZionamento della stessa}

Peculiare è il fatto che il legislatore, a differenza di quanto avviene per il matrimonio all'art. 107 c.c., primo comma ${ }^{21}$, non indichi nella legge n. 76/2016 il momento in cui si perfeziona l'unione civile. L'art. 1, secondo comma, della legge, infatti, recita: «Due persone maggiorenni dello stesso sesso costituiscono un'unione civile mediante dichiarazione di fronte all'ufficiale di stato civile ed alla presenza

${ }^{18}$ Ciò sta a significare, molto brevemente, che una diseguaglianza per esser costituzionalmente legittima, deve essere ragionevole.

19 Sentenza 138/ 2010: «[...] Nell'ambito applicativo dell'art. 2 Cost., spetta al Parlamento, nell'esercizio della sua piena discrezionalità, individuare le forme di garanzia e di riconoscimento delle unioni suddette»; a detto virgolettato, la sentenza 170/2014 aggiunge come sia, ad ogni modo, «riservata alla Corte Costituzionale la possibilità di intervenire a tutela di specifiche situazioni».

${ }^{20}$ Ogni volta che ci riferiremo in questa sede alla legge sopra citata, ci si riferirà sempre alla prima parte della stessa. Non emergerà in analisi, infatti, la seconda parte della legge, quella sulle convivenze, ciò essendo, per così dire, il mero riconoscimento giuridico di una situazione di fatto socialmente rilevante, ma indipendente dal genere sessuale e pertanto poco rilevante in questa sede d'analisi (e che tra l'altro necessiterebbe di ulteriori parametri di comparazione alla luce dell'evoluzione legislativo-giurisprudenziale italiana e spagnola).

${ }_{21}$ Lart. 107, primo comma, c.c. stabilisce che: «nel giorno indicato dalle parti l'ufficiale di stato civile, alla presenza di due testimoni [...]; riceve da ciascuna delle parti personalmente, una dopo l'altra, la dichiarazione che esse si vogliono prendere rispettivamente in marito e moglie, e di seguito dichiara che esse sono unite in matrimonio». 
di due testimoni». Rimane dubbio e non esplicitato quindi -a differenza di quanto avviene nell'articolo del codice ivi sopra citato, ove si esplicita che il momento del perfezionamento del matrimonio è da rinvenire nella dichiarazione dell'ufficiale di stato civile successiva alle rispettive dichiarazioni dei nubendi- il momento in cui l'unione è perfezionata ${ }^{22}$. Non rispondono all'esigenza di esplicitare il momento in cui l'unione è perfezionata nemmeno gli altri commi dell'unico articolo della legge che disciplina l'unione civile, i quali si limitano ad indicare che detta unione è certificata dal relativo documento che l'attesta, che spetta all'ufficiale di stato civile la registrazione di detto atto nell'archivio di stato civile e che dalla costituzione di detta unione derivano diritti e doveri reciproci, non specificando però in nessun comma il momento in cui la stessa si perfeziona ${ }^{23}$.

La dottrina, pertanto, si interroga circa la possibilità -alla luce di una legge che in più punti non appare di chiara interpretazione- dell'applicazione alla stessa di un'interpretazione analogica mutuata dalle norme sull'istituto del matrimonio (così come codicisticamente disciplinato). Infatti, se è pur vero che l'art. 1, ventesimo comma $^{24}$, della legge in esame esclude l'applicazione diretta delle norme del codice civile non espressamente richiamate, ciò non pare implicare la preclusione della

${ }^{22}$ A titolo comparatistico, si specifica come nella legislazione spagnola, il código civil specifichi all'art. 61 come il matrimonio produca effetti a partire dalla sua celebrazione, intendendo pertanto la stessa quale momento in cui il matrimonio si perfeziona. Art. 61, c. 1 «El matrimonio produce efectos civile desde su celebración»; il secondo comma specifica ulteriormente che: «Para el pleno reconocimiento de los mismos serà necesaria su inscripción en el Registro Civil». Ad una prima lettura, sulla scorta della redazione dell'articolo, sembrerebbe che con l'iscrizione del matrimonio ci sia un ulteriore effettività/efficacia dello stesso, lettura sorretta anche dal terzo comma: «El matrimonio no inscrito no perjudicará los derechos adquiridos de buena fe por terceraa personas». Dottrina e giurisprudenza sono però unanimi nel considerare la necessità dell'iscrizione solamente ad probationem tantum, vale a dire quale prova verso i terzi dell'esistenza del vincolo giuridico. Per un approfondimento sul punto si cfr.: J. Díez del Corral Rivas, "Comentario al art. 61», Comentarios del Código Civil, tomo I, dirigido por Cándido Paz-Ares Rodríguez, Luis Díez-Picazo Ponce de León, Rodrigo Bercovitz, Pablo Salvador Coderch, Tirant lo Blanch, Madrid, 1993, art. 61, p. 300 e ss.

${ }^{23}$ Art. 1, terzo comma, legge n. 76/2016: «L'ufficiale di stato civile provvede alla registrazione degli atti di unione civile tra persone dello stesso sesso nell'archivio dello stato civile»; Art. 1, nono comma: «L'unione civile tra persone dello stesso sesso è certificata dal relativo documento attestante la costituzione dell'unione, che deve contenere i dati anagrafici delle parti, l'indicazione del loro regime patrimoniale e della loro residenza, oltre ai dati anagrafici e alla residenza dei testimoni»; Art. 1, undicesimo comma: "Con la costituzione dell'unione civile tra persone dello stesso sesso le parti acquistano gli stessi diritti e assumono i medesimi doveri»; non viene mai esplicitato, però, quando si perfeziona la costituzione dell'unione esplicitata.

${ }^{24}$ Art. 1, c.20, 1. n. 76/2016: «Al solo fine di assicurare l'effettività della tutela dei diritti e il pieno adempimento degli obblighi derivanti dall'unione civile tra persone dello stesso sesso, le disposizioni che si riferiscono al matrimonio e le disposizioni contenenti le parole "coniuge», «coniugi» o termini equivalenti, ovunque ricorrono nelle leggi, negli atti aventi forza di legge, nei regolamenti nonché negli atti amministrativi e nei contratti collettivi, si applicano anche ad ognuna delle parti dell'unione civile tra persone dello stesso sesso. La disposizione di cui al periodo precedente non si applica alle norme del codice civile non richiamate espressamente nella presente legge, nonché alle disposizioni di cui alla legge 4 maggio 1983, n. 184. Resta fermo quanto previsto e consentito in materia di adozione dalle norme vigenti; inoltre, l'art. 1, c. 21, prevede: «Alle parti dell'unione civile 
possibilità di applicazione in via analogica delle stesse, ai sensi dell'art. 12, secondo comma, delle disposizioni preliminari al codice civile (avendo lo stesso portata generale, è pacificamente applicabile ogni volta che si tratti di interpretare una legge che non abbia portata eccezionale ovvero temporanea).

Si potrà quindi ricorrere all'applicazione analogica dell'art. 107, primo comma c.c. -in risposta al quesito di cui sopra circa il momento in cui si perfeziona l'unione civile - che disciplina l'atto di matrimonio e identifica nella dichiarazione dell'ufficiale di stato civile il momento in cui lo stesso si perfeziona; con lo stesso metodo interpretativo pare si possa procedere per tutti quegli ambiti dove la legge sia scarna e non sufficientemente esplicita nel disciplinare aspetti da cui non è possibile prescindere (a scopo esemplificativo: l'interprete può, eventualmente, soprassedere sul fatto che la legge sull'unione nulla dica e preveda circa le formalità richieste precedenti l'atto di unione (pubblicazioni), ma non può certamente soprassedere e, quindi, necessita di colmare -attraverso applicazione analogica-il silenzio della legge con riferimento alle modalità e ai requisiti di opposizione all'unione civile -invece regolati per il matrimonio agli artt. 102-104 c.c.

L'applicazione in via analogica delle norme dettate dal codice per l'istituto del matrimonio conosce dei limiti ontologici e formali ${ }^{25}$, da analizzare caso per caso: ad esempio, pare non ci siano limiti all'applicazione analogica dell'art. 111 del codice civile, che disciplina il matrimonio per procura, così come non paiono esserci limiti -sulla scorta della ratio della norma- circa l'applicazione analogica dell'art. 84 c.c. che si riferisce alla possibilità di contrarre matrimonio prima della maggiore età (al ricorrere di determinate circostanze lasciate all'apprezzamento del giudice) e, pertanto, con esso, la possibilità di applicazione -in caso di unione contratta in violazione dell'art. 84 c.c. - dell'art. 117, c. 2, circa l'impugnazione dello stesso al fine di chiederne l'annullamento, ovvero - conseguentemente/alternativamente e in caso ricorrano i presupposti- degli artt. 390ss. c.c. circa l'emancipazione del minore; non si potranno, invece, applicare analogicamente le norme in tema di obblighi derivanti dall'unione civile, essendo state dettate in tema norme ad hoc nella legge n. 76/2016; non sembra, altresì, vi sia spazio per l'applicazione analogica dell'art. 107 nella parte in cui prevede la lettura ai nubendi degli artt. 143, 144, 147 c.c. da parte dell'ufficiale di stato civile, in quanto la legge in esame -nella descrizione della costituzione dell'unione- non pare, in questi termini, lacunosa, bensì semplificata. Ad ogni modo, la mancata previsione della lettura durante la costituzione dell'unione degli articoli sopra citati desta alcune perplessità, essendo ormai pacifico in dottrina e giurisprudenza che la lettura ad alta voce degli stessi da parte di un pubblico

tra persone dello stesso sesso si applicano le disposizioni previste dal capo III e dal capo x del titolo I, dal titolo II e dal capo II e dal capo v-bis del titolo Iv del libro secondo del codice civile».

25 Sull'opportunità di inserire la legge Cirinnà nel codice, anziché disciplinare le unioni civili con legislazione esogena, ovvero sull'opportunità di richiamare direttamente la disciplina del matrimonio per disciplinare dette unioni si confronti: G.Iorio, «Il disegno di legge sulle unioni civili e sulle convivenze di fatto: appunti e proposte sui lavori in corso", in Nuove leggi civ.comm., 5, 2015, p. 1014 e ss. 
ufficiale sottenda un interesse pubblico (quindi generale ed astratto) per l'istituto in quanto tale; se questa è la ratio primaria, se non esclusiva, della lettura in esame, allora tale omissione normativa potrebbe eventualmente - pur essendo chiara e pacifica la lettura dell'art. 3 della Costituzione offertaci dalla Corte Costituzionale con riferimento all'istituto del matrimonio, rispetto ad un istituto che riconosca l'unione omoaffettiva- esporsi al rischio di essere sottoposta a giudizio di costituzionalità per violazione del principio di uguaglianza -sulla scorta dell'irragionevolezza della disparità di trattamento- con riferimento alla dignità ed importanza riconosciuti al nucleo familiare a prescindere dal nomen iuris ad esso attribuito.

E' pacifico come il ricorso al procedimento analogico non possa essere prospettato con riferimento alle norme sull'adozione, essendo indubbio ed esplicito sul punto l'art.1, ventesimo comma, nella sua ultima parte: «La disposizione di cui al periodo precedente non si applica alle norme del codice civile non richiamate espressamente nella presente legge, nonché alle disposizioni di cui alla legge 4 maggio 1983, n. 184. Resta fermo quanto previsto e consentito in materia di adozione dalle norme vigenti ${ }^{26}$.

Infine, si sottolinea come la legge n.76/2016, oltre a non richiedere l'adempimento di formalità precedenti la costituzione del vincolo (si pensi qui alle pubblicazioni matrimoniali), nulla dispone circa l'eventuale possibilità di opposizione all'unione civile. Si può quindi, in questa ulteriore circostanza, ipotizzare -sulla base della ratio sui cui si fonda detto istituto- la possibilità di applicazione analogica delle norme codicistiche (artt. 102-104 c.c.) che lo regolamentano. La disciplina, infatti, pur essendo legata da un punto di vista formale alla disciplina sulle pubblicazioni, fonda la sua ratio, ex art. 102 c.c., nella possibilità -per i terzi di cui agli articoli sopracitati- di poter impedire il matrimonio tra due persone in presenza di condizioni ostative; esigenza, quindi, che sussiste anche in caso di unione civile.

\section{CAUSE DI IMPEDIMENTO ALla COSTITUZIONE DI UNIONE CIVILE E CAUSE DI NU- LLITÀ DELla STESSA: DifFERENZE E ANALOGIE CON L'ISTITUTO DEL MATRIMONIO}

Con riferimento alle cause di impedimento alla costituzione di un'unione civile, il legislatore non ha scelto la strada più sistematica del richiamo -in quanto compatibile- delle norme codicistiche sul matrimonio rubricate "condizioni necessarie per contrarre matrimonio ${ }^{27}$, ma ha, invece, espressamente stabilito e tipizzato dette cause impeditive all'art. 1 c. 4, della legge n. 76/2016 (pur essendo detta norma, da un punto di vista contenutistico, pressoché un duplicato di quanto già previsto nel codice circa le cause impeditive nella disciplina matrimoniale).

${ }^{26}$ Come già specificato in nota nel precedente paragrafo, si analizzerà in altro paragrafo la problematica delle adozioni da parte delle coppie same-sex e i limiti alla stessa, in chiave comparativa con la legislazione spagnola e a quanto esplicitato dal Tribunal Costitucional nel 2012.

${ }_{27}$ Libro I, Titolo VI, capo III, sezione I del Codice civile. 
Senza ora entrare nel dettaglio della norma ivi sopra descritta, rileva sottolineare altresì come, in ottica comparata con la disciplina prevista dal Codice civile circa l'istituto del matrimonio, l'art. 1 , c. $5^{28}$, preveda che all'unione civile si applichino gli art. 65 e 68 c.c. -nonché le disposizioni di cui agli articoli 119, $120,123,125,126,127,128,129$ e 129-bis del codice civile-; da qui, l'art. 1 c. 6, dispone che: "Lunione civile costituita in violazione di una delle cause impeditive di cui al comma 4, ovvero in violazione dell'articolo 68 del codice civile, può essere impugnata da ciascuna delle parti dell'unione civile, dagli ascendenti prossimi, dal pubblico ministero e da tutti coloro che abbiano per impugnarla un interesse legittimo e attuale. L'unione civile costituita da una parte durante l'assenza dell'altra non può essere impugnata finche' dura l'assenza ${ }^{29}{ }^{\prime}$.

La norma non pare correttamente formulata, in quanto la norma di cui all'art. 68 c.c. non contiene prescrizioni violabili per il tramite della costituzione di unione civile; la norma di cui sopra è, quindi, da intendersi in combinato con il comma precedente -che dispone per l'unione civile l'applicazione diretta dell'art. 65 c.c.-. Pertanto, l'unione civile costituita ai sensi dell'art. 65 c.c. (nuova unione costituita dopo che la sentenza di dichiarazione di morte presunta del precedente unito sia divenuta eseguibile) è da considerarsi nulla ogni qualvolta che la persona di cui sia stata dichiarata la morte presunta torni ovvero sia accertata la sua esistenza.

Rileva evidenziare come il legislatore non abbia contemplato nella disciplina altre due fattispecie:

- il caso di persona sposata che contragga unione civile in seguito a dichiarazione di morte presunta del coniuge, ovvero il caso di persona che abbia contratto unione civile e in seguito a dichiarazione di morte presunto del civilmente unito, si sposi.

- Invero, anche in siffatte fattispecie, per colmare il vuoto normativo è possibile ipotizzare l'applicazione analogica degli artt. 65 e 68 c.c., quale argomento a contrario sulla scorta di quanto, invece, è espressamente previsto con riferimento alle cause impeditive alla costituzione di unione civile (art. 1, c. 4 , lett. a) 1. n. 76/2016) ${ }^{30}$.

- In conclusione, con riferimento alle cause di nullità dell'unione civile, urge evidenziare quanto espressamente previsto all'art. 1, c. 7, della legge in esame ${ }^{31}$,

${ }^{28}$ Art. 1, c. 5, 1. n. 76/2016 «La sussistenza di una delle cause impeditive di cui al comma 4, comporta la nullità dell'unione civile tra persone dello stesso sesso. All'unione civile tra persone dello stesso sesso si applicano gli articoli 65 e 68, nonché le disposizioni di cui agli articoli 119, 120, $123,125,126,127,128,129$ e 129-bis del codice civile».

${ }^{29}$ Lultima parte di detta norma ricalca il contenuto dell'art. 117 c. 3, c.c, con riferimento al matrimonio.

${ }^{30}$ Art. 1, c. 4, lett. a) 1.n. 76/2016: «Sono cause impeditive per la costituzione dell'unione civile tra persone dello stesso sesso: a) la sussistenza, per una delle parti, di un vincolo matrimoniale o di un'unione civile tra persone dello stesso sesso".

31 Art. 1, c. 7, 1. n.76/2016: "L'unione civile può essere impugnata dalla parte il cui consenso è stato estorto con violenza o determinato da timore di eccezionale gravità determinato da 
e in particolare il richiamo che detto comma attua dell'art. 122 c. 3, c.c. (esso richiama, di detto comma, solo i punti 2, 3 e 4).

Orbene, detto articolo 122, c.c., sancisce:

«Il matrimonio può essere impugnato da quello dei coniugi il cui consenso è stato estorto con violenza o determinato da timore di eccezionale gravità derivante da cause esterne allo sposo.

Il matrimonio può altresì essere impugnato da quello dei coniugi il cui consenso è stato dato per effetto di errore sull'identità della persona o di errore essenziale su qualità personali dell'altro coniuge.

L'errore sulle qualità personali è essenziale qualora, tenute presenti le condizioni dell'altro coniuge, si accerti che lo stesso non avrebbe prestato il suo consenso se le avesse esattamente conosciute e purché l'errore riguardi:

1) l'esistenza di una malattia fisica o psichica o di un'anomalia o deviazione sessuale, tali da impedire lo svolgimento della vita coniugale;

2) l'esistenza di una sentenza di condanna per delitto non colposo alla reclusione non inferiore a cinque anni, salvo il caso di intervenuta riabilitazione prima della celebrazione del matrimonio. L'azione di annullamento non può essere proposta prima che la sentenza sia divenuta irrevocabile;

3) la dichiarazione di delinquenza abituale o professionale;

4) la circostanza che l'altro coniuge sia stato condannato per delitti concernenti la prostituzione a pena non inferiore a due anni. Lazione di annullamento non può essere proposta prima che la condanna sia divenuta irrevocabile;

5) lo stato di gravidanza causato da persona diversa dal soggetto caduto in errore, purché vi sia stato disconoscimento ai sensi dell'art. 233, se la gravidanza è stata portata a termine.

L'azione non può essere proposta se vi è stata coabitazione per un anno dopo che siano cessate la violenza o le cause che hanno determinato il timore ovvero sia stato scoperto l'errore».

Dal richiamo legislativo all'articolo 122 , c. 3, c.c., ivi sopra riportato, è stato omesso il richiamo dei punti 1) e 5); se può apparire comprensibile la ragione per cui possa esser stato espunto quest'ultimo numero - partendo dalla considerazione che

cause esterne alla parte stessa. Può essere altresì impugnata dalla parte il cui consenso è stato dato per effetto di errore sull'identità della persona o di errore essenziale su qualità personali dell'altra parte. L'azione non può essere proposta se vi è stata coabitazione per un anno dopo che è cessata la violenza o le cause che hanno determinato il timore ovvero sia stato scoperto l'errore. L'errore sulle qualità personali è essenziale qualora, tenute presenti le condizioni dell'altra parte, si accerti che la stessa non avrebbe prestato il suo consenso se le avesse esattamente conosciute e purché l'errore riguardi: a) l'esistenza di una malattia fisica o psichica, tale da impedire lo svolgimento della vita comune; b) le circostanze di cui all'articolo 122, terzo comma, numeri 2), 3) e 4), del codice civile. 
una coppia omosessuale non può, mediante mero accoppiamento sessuale, procreare un figlio-, non si comprende la ragione dell'espunzione del numero 1) dal richiamo del comma in esame.

La norma di cui al punto 1 , come evidenziato in dottrina, si riferisce a tutti quei comportamenti, alle menomazioni fisiche ovvero a tutti quegli atteggiamenti psicologici patologicamente anomali riferibili alla sfera sessuale, in grado di interferire irreversibilmente con la vita sessuale di una coppia, come il sadismo ovvero la ninfomania ${ }^{32}$.

Pur non volendo apparire incauti, l'omesso richiamo al punto 1 della norma di cui all'art. 122, c. 3, c.c., pare passibile di esposizione a censura di incostituzionalità, stante l'apparente carattere discriminatorio della norma: non si comprende, infatti, quale possa essere il criterio di ragionevolezza applicato a questa diversità di trattamento con riferimento alle cause di nullità da riferirsi ai due istituti (matrimonio e unione civile) ai sensi e nei modi previsti dall'art. 3 Cost., secondo cui le patologie sopra esposte possano esser causa di nullità della costituzione di matrimonio, ma non già di unione civile.

Proseguendo nella disamina della legge di recente introduzione, menzione specifica merita l'art. 10, c.10, 1. n. 76/2016: «Mediante dichiarazione all'ufficiale di stato civile le parti possono stabilire di assumere, per la durata dell'unione civile tra persone dello stesso sesso, un cognome comune scegliendolo tra i loro cognomi. La parte può anteporre o posporre al cognome comune il proprio cognome, se diverso, facendone dichiarazione all'ufficiale di stato civile» (quanto disposto in quest'ultima parte della norma può verificarsi solo in caso le due persone costituenti l'unione abbiano cognome diverso).

Tale disposizione risponde indubbiamente ad una visione più moderna della disciplina sul cognome familiare, che in questa sede ci si auspica possa influenzare un dibattito politico (già esistente) circa la modifica dell'art. 143 bis c.c., figlio di un orientamento superato, decisamente poco coerente con l'affermazione della parità -formale e sostanziale- dei coniugi all'interno del nucleo costituito.

Pur se, in seguito, ci sarà modo di evidenziare altri punti critici della legge, potenzialmente passibili di censura costituzionale, è opportuno evidenziare come alla norma prevista sulla scelta del cognome comune, si possa attribuire valore simbolico, oltre che normativo: tale possibilità attribuisce agli uniti civilmente di scegliere e stabilire un "cognome familiare», riconoscendo così agli stessi la possibilità di esser riconosciuti, normativamente e socialmente, come nucleo e non già solamente come relazione omoaffettiva tra due persone giuridicamente rilevante. Proseguendo in questo senso rileva, però, sottolineare -in tema di cognome familiare-, il vuoto legislativo contenuto nella legge n. 76/2016, laddove non abbia espressamente richiamato i commi terzo e quarto dell'art. 5, legge n. 898/70 rubricata «Disciplina dei casi scioglimento del matrimonio».

32 Per un approfondimento ricognitivo dei caratteri tipici di cosa possa essere definito deviazione sessuale, si cfr. sentenza Cass. n. 3407/2013. 
Essi stabiliscono, rispettivamente, che: «Il tribunale, con la sentenza con cui pronuncia lo scioglimento o la cessazione degli effetti civili del matrimonio, può autorizzare la donna che ne faccia richiesta a conservare il cognome del marito aggiunto al proprio quando sussista un interesse suo o dei figli ${ }^{33}$ meritevole di tutela» e «La decisione di cui al comma precedente può essere modificata con successiva sentenza, per motivi di particolare gravità, su istanza di una delle parti».

Orbene, dato il contenuto ontologico del cognome familiare ivi sopra esplicitato, non si comprende come, nei casi in cui sussista un interesse meritevole di tutela, non sia da considerare discriminatorio -e pertanto eventualmente costituzionalmente censurabile- la non estensione -anche eventualmente analogica- della possibilità di conservare il cognome scelto ai sensi dell'art. 1, c. 10, della legge n. 76/2016 ${ }^{34}$.

Continuando in ottica comparativa con l'istituto del matrimonio e la disciplina codicistica e legislativa che lo riguarda, la legge n. 76/2016 stabilisce piena equiparazione dell'unione civile e dell'istituto del matrimonio con riferimento al regime patrimoniale; non così, invece, con riferimento agli obblighi per i contraenti derivanti dalla costituzione di unione civile.

Infatti, la legge in esame sulle unioni civili, pur ricalcando - da un punto di vista contenutistico- per la maggior parte le norme codicistiche in tema di diritti e doveri reciproci dei coniugi (comprese quelle concernenti gli alimenti) ${ }^{35}$, non ha

33 Ad abundantiam, si evidenzia come la disciplina italiana, con riferimento alla scelta del cognome familiare, sia superata e necessiti di intervento legislativo. A titolo esemplificativo, si cfr. Corte Edu, sez. II, 77/2014: «La normativa italiana che assegna ai figli legittimi il cognome del genitore di sesso maschile viola l'art. 14 della convenzione per la salvaguardia dei diritti dell'Uomo e delle libertà fondamentali, in combinato disposto con l'art. 8 della stessa convenzione; la normativa predetta va, quindi, modificata». In comparazione, invece, con la disciplina spagnola, quest'ultima è decisamente più avanzata e in linea con la normativa europea e le politiche di genere: in Spagna vige, infatti, la regola del «doppio cognome», per cui ogni figlio porta il primo cognome di entrambi i genitori, nell'ordine deciso in accordo tra di essi( in caso di disaccordo, è attribuito al figlio il primo cognome del padre insieme al primo cognome della madre; raggiunta la maggiore età, lo stesso può proporre istanza per invertire l'ordine dei cognomi). In caso di adozione omogenitoriale ovvero di riconoscimento del figlio naturale da parte di uno solo dei genitori, lo stesso prenderà i due cognomi del genitore.Si cfr. sul punto art. 109 del Codigo Civil (modificato dalla l. n. 40/99) e gli artt. 5356 de la Ley sobre el Registro Civil del 1957 -si specifica come già prima della modifica del '99, i cittadini spagnoli da sempre hanno il primo cognome del padre e il primo cognome della madre, rispettivamente in quest'ordine-.

${ }^{34}$ Con riferimento a siffatta fattispecie, essendo il cognome uno degli elementi identificativi della personalità dell'individuo, di cui alcuni aspetti potrebbero essere stati acquisiti, ovvero consolidati, durante il tempo e a causa dell'unione, si potrebbe ipotizzare, -alla luce del vuoto legislativo e in caso non si applichino per analogia i commi 3 e 4 dell'art. 5 della legge n. 898/70- un'azione giudiziaria a tutela del diritto al nome ai sensi degli art. 2 Cost. e 7 c.c. Si cfr. sul punto: AA. VV., Unioni civili e convivenze di fatto L. 20 maggio 2016, n. 76, a cura Marilena Gorgoni, Maggioli, 2016, p. 111 e ss.

35 Art. 1, c. 19, 1. n. 76/2016: «All'unione civile tra persone dello stesso sesso si applicano le disposizioni di cui al titolo xıI del libro primo del codice civile, nonché gli articoli 116, primo comma, 146, 2647, 2653, primo comma, numero 4), e 2659 del codice civile. 
previsto, per gli uniti civilmente, gli obblighi reciproci di fedeltà e di collaborazione nell'interesse della famiglia ${ }^{36}$ (previsti dall'art. 143, c. 2, c.c.).

Si provi, pertanto, in questa sede, a comprendere le ragioni implicite (discriminatorie?) celate dietro a tale scelta, e a quali conseguenze può condurre tale vuoto legislativo.

L'intento del legislatore pare esser quello -quantomeno nell'ambito di analisi di cui ora ci si andrà ad occupare- di tenere su due livelli distinti l'istituto del matrimonio e quello dell'unione civile, dove solo dal primo pare nascere una vera e propria famiglia; questo è ciò che pare emergere da un analisi del dato letterale di quanto trasposto nell'art. 1, c. 11, della legge n. 76/2016 di ciò che è contenuto nell'art. 143 c.c.: se qui, infatti, si parla di «bisogni della famiglia», nella norma di cui al comma 11 -che ricalca pressoché pedissequamente il testo dell'art. 143 c.c.- pare esser frutto di una puntuale scelta legislativa (e non di una «svista») il fatto che si parli solamente di «bisogni comuni», senza menzionare mai, all'interno del dettame legislativo di detto comma, il termine "famiglia», che, invece, nel testo dell'articolo 143 c.c. di cui sopra, compare ben due volte ${ }^{37}$.

Scelta che il legislatore persegue (parzialmente) anche nel comma successivo: se è pur vero, infatti, che in questo comma sancisce che l'unione civile vada a costituire una "vita familiare», è pur vero che, nella trasposizione in detta norma dell'art. 144 c.c., tralascia di utilizzare il termine "famiglia» ogni qualvolta nello stesso venga utilizzato, omettendolo o sostituendolo nuovamente con l'aggettivo «comune» ${ }^{38}$.

36 Obbligo che sfugge ad un rigido inquadramento, e del cui contenuto non è semplice fornire una cornice. Su detto obbligo, due orientamenti opposti: l'uno che lo considera mera ripetizione, per così dire riassuntiva, degli obblighi imposti ai coniugi (si cfr. S. Alagna, Famiglia e rapporti tra coniugi nel nuovo diritto, Milano, Giuffrè, 1985, p. 154 e ss.); l'altro che, invece, considera l'obbligo di collaborazione familiare quale obbligo autonomo, contenente un quid pluris rispetto a quanto previsto dagli altri obblighi codicisticamente previsti per i coniugi, essendo lo stesso da riferire alle esigenze della famiglia considerata nella sua unità (M.Paradiso, "I rapporti personali tra coniugi, artt. 143-148", in Comm. Schlesinger, Milano, Giuffrè, 1990, p. 49 e ss.). Lo stralcio potrebbe essere quindi dovuto, nel primo caso, alla volontà di non reiterare un'inutile ripetizione; nell'altro caso, in coerenza con quanto finora sostenuto e sulla scorta delle scelte legislative finora analizzate, tale stralcio sarebbe, in ottica sistematica, l'ennesima eliminazione di ogni riferimento al concetto di «famiglia», quale nucleo emergente dalla costituzione di unione civile.

${ }^{37}$ Art. 143 c.c. «Con il matrimonio il marito e la moglie acquistano gli stessi diritti e assumono i medesimi doveri. Dal matrimonio deriva l'obbligo reciproco della fedeltà, all'assistenza morale e materiale, alla collaborazione nell'interesse della famiglia e alla coabitazione. Entrambi i coniugi sono tenuti, ciascuno in relazione alle proprie sostanze e alla propria capacità di lavoro professionale o casalingo, a contribuire ai bisogni della famiglia»; si cfr. con: Art. 1, c. 11, 1. n. 76/2016: «Con la costituzione dell'unione civile tra persone dello stesso sesso le parti acquistano gli stessi diritti e assumono i medesimi doveri; dall'unione civile deriva l'obbligo reciproco all'assistenza morale e materiale e alla coabitazione. Entrambe le parti sono tenute, ciascuna in relazione alle proprie sostanze e alla propria capacità di lavoro professionale e casalingo, a contribuire ai bisogni comuni».

38 Art.1, c. 12, 1. n. 76/2016: «Le parti concordano tra loro l'indirizzo della vita familiare e fissano la residenza comune; a ciascuna delle parti spetta il potere di attuare l'indirizzo concordato"; si cfr. con: Art.144 c.c.: «I coniugi concordano tra loro l'indirizzo della vita familiare e fissano la 
Tanto premesso, la ragione di tale atteggiamento legislativo pare ravvisabile solo in termini "discriminatori», espressione - pare- della volontà (schizofrenica, dato l'uso dell'aggettivo "familiare», ma non già della parola "famiglia», come a voler indicarne significati differenti) di non voler definire "famiglia»" ${ }^{39}$ quanto costituito mediante un'unione omoaffettiva.

Una giustificazione a tale decisione politica non sembra ravvisabile nemmeno ipotizzando che la stessa sia figlia della ricerca di coerenza, continuità e sistematicità con lo stralcio dalla legge n. 76/2016 della «Stepchild Adoption», essendo in dottrina e giurisprudenza pacifico che l'istituto del matrimonio non è ristretto né vincolato alla capacità coeundi e generandi dei nubendi, salvo quanto disposto dall'art. 122, c. 2 e c. 3 n. 1), c.c ${ }^{40}$ (a meno che non si voglia interpretare l'omesso richiamo, precedentemente evidenziato, nella legge n. 76/2016, del punto 1) del terzo comma dell'art. 122 c.c., in combinato con l'omissione ripetuta in detta legge del termine "famiglia» quale conseguenza alla costituzione di unione civile, come a voler intendere che nell'idea del legislatore, del ' 42 prima, e del ' 75 poi (riforma del diritto di famiglia), si volesse intendere che il termine «famiglia» sia da ascrivere solo a coppie potenzialmente in grado di generare vita, e che, quindi, il termine "famiglia» plurimamente diffuso e profuso nelle norme sul matrimonio non sia da riferire a quelle coppie che decidono di contrarre matrimonio pur difettando l'uno, l'altro, ovvero entrambi, di capacità coeundi ovvero generandi, costituendo le stesse eccezione/deviazione dallo modello tipico normativamente disciplinato.

In altri termini; le coppie omoaffettive non possono, da un punto di vista meramente biologico-naturale, generare vita e, pertanto, parrebbe che il legislatore non voglia considerare con il termine "famiglia» ciò che consegue all'unione civile delle stesse, negando alle stesse altresì la possibilità di annullamento dell'unione ai

residenza della famiglia secondo le esigenze di entrambi e quelle preminenti della famiglia stessa. A ciascuno dei coniugi spetta il potere di attuare l'indirizzo concordato».

39 Per una visione del concetto di famiglia in chiave «moderna», un archetipo modificato ed evolutosi con realtà sociale: F. Gazzoni, «Dal concubinato alla famiglia di fatto», Giuffrè, Milano, 1983, p. 151 e ss.; L. Balestra, «L’evoluzione del diritto di famiglia e le molteplici realtà affettive», in Riv. Trim. Dir. Proc. Civ., Giuffrè , 2011, 1105 e segg., spec. 1115-1116.

${ }^{40}$ Il diritto civile italiano non considera l'impotenza, sia coeundi che generandi, un impedimento al matrimonio, salvo che il consenso allo stesso sia viziato da errore essenziale su qualità personali dell'altro coniuge. L'impotenza può pertanto divenire motivo di invalidità in caso sia ignorata dall'altro coniuge e qualora quest'ultimo ritenga che detta qualità sia da considerare errore essenziale viziante il consenso. Detto errore, ai sensi dell'art. 122 c. 3, si considera «essenziale qualora, tenute presenti le condizioni dell'altro coniuge, sia accertato giudizialmente che lo stesso non avrebbe prestato il suo consenso se le avesse esattamente conosciute e purché l'errore riguardi, tra gli altri: L'esistenza di una malattia fisica o psichica o di una anomalia o deviazione sessuale, tali da impedire lo svolgimento della vita coniugale [...]». Per un approfondimento sul punto si veda inoltre: A. Figone, «Sulla nullità del matrimonio per impotenza a generare, nota a Corte d'Appello Napoli, 13 aprile 1995», V, in Famiglia e diritto, IPSOA, 1996, I, p. 340 e ss. 
sensi del punto 1) c. 3, dell'art. 122 c.c. (che coinvolge non solo l'impotenza, ma, invero, anche le deviazioni e le patologie psicologico-sessuali ${ }^{41}$ ).

\section{Obbligo di fedeltà: la mancata pRevisione dello Stesso PeR GLi Uniti CIVILMENTE}

Pur non essendo mai stati esplicitati i motivi dell'omissione dell'obbligo di fedeltà dai doveri degli uniti civilmente, questa non può esser considerata una mera dimenticanza del legislatore, essendo invece detto obbligo previsto nel disegno di legge e successivamente stralciato, così come l'istituto della stepchild adoption, ai fini dell'approvazione della legge che li conteneva.

Pur non volendo considerare i due stralci alla legge collegati tra loro, è pacifico che il filo invisibile che gli stessi ricollega è da rinvenire nel tipo di relazione che il legislatore pare consideri sussistere alla base dell'unione civile: una relazione meno vincolante, e forse, anche (o solo?) per questo, inadatta a ricreare quell'ambiente familiare stabile necessario ai fini dell'esito positivo di una richiesta di adozione, che si basa esclusivamente sul soddisfacimento degli interessi del minore, per il suo pieno e libero sviluppo ${ }^{42}$.

Se, pertanto, il significato della mancata previsione dell'obbligo di fedeltà sia da ascrivere ad una aprioristica (e non adeguatamente motivata) presa di posizione legislativa che rinvenga ex se in un'unione omoaffettiva una relazione meno vincolante, si esporrebbe l'art. 1, c. 11, l. n.76/2016, a censura di incostituzionalità, -nella parte in cui non prevede l'obbligo di fedeltà- in quanto quello di cui sopra, se non motivato altrimenti, costituirebbe assunto discriminatorio.

E' stato sostenuto da alcuni parlamentari (e depositato in un d.d.l. di una sola riga il 25 febbraio del 2016, data precedente all'approvazione della legge $\mathrm{n}$. $76 / 2016$, che risale, come già più volte specificato, al mese di maggio), che tale previsione, lungi dall'essere discriminatoria, è, anzi, un modello da seguire ${ }^{43}$; il

${ }_{41}$ Non si vuole prendere in considerazione in questa sede la possibilità che il legislatore non abbia previsto la possibilità di annullamento dell'unione civile in caso di errore sulla deviazione sessuale del partner per il fatto di aver catalogato l'omosessualità stessa quale deviazione da un comportamento e orientamento sessuale prevalente, in quanto ciò sarebbe contrario ai diritti sanciti nella Carta Costituzionale, oltre che nelle normative comunitarie e nei Trattati internazionali.

${ }^{42}$ Ricordiamo che il diritto italiano e comunitario, a prescindere dall'orientamento sessuale dell'adottante/adottanti, non prevede un diritto ad essere genitori (pur prevedendo la generale libertà di autodeterminarsi, da contemperare con altri interessi di pari rango [...] sentenza della C. Cost. n. 152/2014, si veda infra); bensì, prevede un diritto del minore ad avere una famiglia: si cfr. artt. $30 \mathrm{e}$ 31, Cost., legge n. 184/83 (e successive modifiche: 1. n. 149/2001, che recepisce i principi contenuti nella Convenzione sulla tutela dei diritti dell'infanzia del 1989), Carta dei diritti fondamentali dell'Unione Europea, art. 24.

43 In Spagna, il dovere di fedeltà, seppur previsto, ha perso significato giuridico (e sociale) non potendo più essere, oggi, causa di addebito della separazione. La riforma del 2005, infatti, modifica la disciplina della separazione e del divorzio, introducendo il divorzio senza causa, eliminando cos', 
risultato di una legge «moderna», contro, invece, una disciplina ormai obsoleta a fronte dell'equiparazione -con legge n. 219 del 2012- tra figli naturali e legittimi e del cambiamento dei costumi sessuali.

Ad abundantiam, si specifica come tale d.d.l -il cui contenuto, come prima specificato, è di una sola riga, che così recita: «Togliere dall'articolo 143 del Codice Civile il riferimento all'obbligo reciproco di fedeltà tra i coniugi»-sia stato proposto nella medesima seduta in cui è stato discusso il maxi-emendamento (poi approvato) al d.d.l Cirinnà, oggi divenuto, con dette modifiche, legge n. 76/2016.

Ora, pur non volendo cedere a dietrologie, il ragionamento pare quantomeno contradditorio.

In giurisprudenza è ormai pacifico che l'obbligo di fedeltà non è più strettamente legato alla fedeltà sessuale, bensì esso coinvolge il più ampio dovere di lealtà; la violazione dell'obbligo di fedeltà può oggi esser persino slegata dalla consumazione carnale, potendo aver valore giudiziale finanche una relazione amorosa platonica ${ }^{44}$. La Cassazione ha definito il dovere di fedeltà come l'obbligo che "consiste nell'impegno, ricadente su ciascun coniuge, di non tradire la fiducia reciproca ovvero di non tradire il rapporto di dedizione fisica e spirituale tra i coniugi che dura quanto dura il matrimonio e non deve essere intesa soltanto come astensione da

di fatto, il dovere di fedeltà da un punto di vista sostanziale, non essendo più possibile sanzionare il medesimo quale motivo di addebito della separazione (rileva oggi solo come eventuale motivo di diseredazione). Detta riforma prevede, in tema di scioglimento del vincolo, la medesima disciplina prevista in Italia per le Unioni civili: domanda unilaterale, senza necessità di motivazione della stessa, e il decorrere di 3 mesi a partire dal momento della richiesta al fine di ottenere lo scioglimento del vincolo (matrimoniale in Spagna, di unione civile in Italia. Se da un lato quindi, si ravvisa nella legge Cirinnà un allineamento con il resto d'Europa per modalità e tempistiche dello scioglimento del vincolo, d'altro canto si ravvisa (un'ennesima e immotivata) differenza di trattamento passibile di censura costituzionale, rispetto a quanto previsto per l'istituto del matrimonio dalla legge italiana sul divorzio n. 898/70 (e successive riforme). Se è pur vero che nel 2015 sono stati rivisti (in diminutio) i tempi necessari all'ottenimento del divorzio in seguito a separazione, è pur sempre vero che detti tempi e le modalità di scioglimento del vincolo non sono i medesimi richiesti per lo scioglimento del vincolo di unione civile (per lo scioglimento del matrimonio è necessario un tempo di separazione di 6 mesi, che "attenua" ma non elimina il vincolo matrimoniale; separazione non richiesta per l'ottenimento dello scioglimento dell'unione civile; è inoltre prevista, per gli uniti civilmente, la possibilità di «divorzio rapido» ai sensi degli artt. 6 e 12 della legge 64/2004). Si veda sul punto: Gilda Ferrando, Diritto di famiglia, Zanichelli, 2013, inserto di aggiornamento settembre 2016 dal titolo: «Unioni civili e convivenze» pubblicato da Zanichelli, Torino, 2016. Ad abundantiam, a titolo comparatistico e con riferimento al precedente evidenziato dovere di fedeltà, rileva sottolineare come l'equiparazione giuridica tra figli naturali e legittimi in Spagna sia avvenuta nel 1981, 31 anni prima che in Italia. Per un approfondimento di queste tematiche si cfr: G. García Cantero, in Castán Tobeñas, Derecho civil español, t. 5-II, Madrid, 1995; C. Martinez de Aguirre Aldaz, P. de Pablo Contreras, M. Pérez Álvarez, Curso de derecho civil, volumen IV, Derecho de Familia, 5. ${ }^{a}$ edición, Madrid 2016, p. 326 e ss.

44 Sul punto, Cass. 8929/2013: «la relazione di un coniuge con estranei rende addebitabile la separazione, ai sensi dell'art. 151 c.c., non solo quando si sostanzi in un adulterio ma anche quando, in considerazione degli aspetti esteriori con cui è coltivata e dell'ambiente in cui i coniugi vivono, dia luogo a plausibili sospetti di infedeltà e comporti offesa alla dignità e all'onore dell'altro coniuge [...]». 
relazioni sessuali extraconiugali. La violazione dell'obbligo di fedeltà può assumere rilievo anche in assenza della prova specifica di una relazione sessuale extraconiugale intrapresa da un coniuge, essendo sufficiente l'esternazione di comportamenti tali da ledere il rapporto di dedizione fisica e spirituale tra i coniugi ferendo la sensibilità e la dignità di colui o colei che subisce gli effetti di quei comportamenti. Ciò può avvenire anche dopo l'insorgere dello stato di separazione non essendo da escludere che questa lasci sussistere tra i coniugi una (magari limitata) solidarietà, tale da giustificare la permanenza dell'obbligo di fedeltà [...] $»^{45}$.

Detto dovere, pertanto, lungi dall'essere obsoleto, si è nel corso del tempo modificato (radicalmente con la riforma del diritto di famiglia del 1975, che ha novellato gli artt. 143-144-145 c.c., e poi, successivamente, gradualmente, alla luce dell'evoluzione giurisprudenziale) fino a diventare qualcosa di più complesso e ampio del mero dettame letterale che detto dovere prevede: «la nozione di fedeltà coniugale va avvicinata a quella di lealtà, la quale impone di sacrificare gli interessi e le scelte individuali di ciascun coniuge che si rivelino in conflitto con gli impegni e le prospettive della vita comune [...]. In questo quadro la fedeltà affettiva diventa componente di una fedeltà più ampia che si traduce nella capacità di sacrificare le proprie scelte personali a quelle imposte dal legame di coppia e dal sodalizio che su di esso si fonda» ${ }^{46}$.

Sulla scorta di quanto finora esplicitato, non si comprende pertanto come detto dovere possa essere considerato vetusto e simbolo di un retaggio culturale superato. Pare, anzi, elemento cardine e fondante del funzionamento del matrimonio, così come ontologicamente definito. Parrebbe pertanto opportuno che detto dovere non solo non sia eliminato dalla previsione dell'art. 143 c.c., bensì esteso agli uniti civilmente. Eventualmente, sulla base dell'evoluta interpretazione giurisprudenziale dello stesso, si potrebbe auspicare un intervento legislativo al fine di sostituire il dovere

45 Cass. n. 9287/1997.

46 Così, Cass. n. 15557/2008. Ad abundantiam, si consideri che qualsiasi violazione degli obblighi di cui all'art. 143, per essere fonte di addebito di separazione dev'essere causa e non conseguenza della crisi matrimoniale, e le singole violazioni dei partner sono da valutare complessivamente. Sul punto si cfr., ex multis,: - Cass. 6697/2009: «Il giudice del merito non può fondare la pronuncia di addebito della separazione sulla mera inosservanza, da parte di uno dei coniugi, dei doveri di cui all'art. 143 c.c., ma deve verificare la effettiva incidenza delle relative violazioni, nel determinarsi della situazione di intollerabilità della convivenza [...] Di fronte ad un comportamento contrario ai doveri del matrimonio da parte di entrambi i coniugi, la condotta dell'uno non può essere giudicata senza un suo raffronto con quella dell'altro, perché solo tale comparazione consente di riscontrare se e quale incidenza le stesse abbiano rivestito, nel loro reciproco interferire, nel verificarsi della crisi coniugale [...]»; - Cass. 27730/2013: «In tema di separazione personale tra coniugi, il giudice non può fondare la pronuncia di addebito sulla mera inosservanza dei doveri di cui all'art. 143 cod. civ., dovendo, per converso, verificare l'effettiva incidenza delle relative violazioni nel determinarsi della situazione di intollerabilità della convivenza. [...] In particolare, l'inosservanza dell'obbligo di fedeltà coniugale in tanto può giustificare l'addebito della separazione al coniuge responsabile, in quanto determini la situazione d'intollerabilità del protrarsi della convivenza coniugale ma non anche se intervenga dopo che questa situazione sia già maturata e dunque in un contesto di disgregazione della comunione materiale e spirituale $[\ldots]$ ». 
di fedeltà, nel suo tenore letterale, con il più puntuale ed ampio dovere di lealtà: un intervento legislativo, quindi, non già in chiave eliminatoria, bensì modificativa ed ampliativa; la previsione di un dovere di lealtà, ancor più ontologicamente complesso, complessivo e totalizzante rispetto al tenore letterale del dovere di fedeltà (la cui interpretazione giurisprudenziale deriva non già da mera interpretazione letterale, ma anche e soprattutto da interpretazione sistematica ed estensiva).

La non previsione del dovere di fedeltà (ovvero la non previsione di un dovere di lealtà, che lo stesso contenga) per gli uniti civilmente appare, pertanto, non solo formalmente discriminatoria, ma anche sostanzialmente discriminatoria: l'unito civilmente non può ottenere tutela avverso il partner, né quest'ultimo sarà sanzionato in caso di violazione di detto obbligo, giacché non previsto per gli uniti civilmente dalla legge n. 76/2016.

L'unica tutela invocabile per il contraente dell'unione civile rimane, pertanto, ove ne sussistano i presupposti, quella aquiliana, ex art. 2043 c.c. (dato il presupposto necessario al verificarsi della fattispecie, consistente nel danno ingiusto -seppur patrimoniale o non patrimoniale-si restringe - di molto-il ventaglio delle fattispecie sanzionabili) ${ }^{47}$.

Non solo un numero inferiore di fattispecie tutelate in caso di separazione con addebito per gli uniti civilmente rispetto a coloro che hanno contratto matrimonio, ma anche tempi e modalità di scioglimento dell'unione differenti rispetto al matrimonio. L'art. 1, c. 24, l. n. 76/2016 prevede, infatti, che: «L'unione civile si scioglie, inoltre, quando le parti hanno manifestato anche disgiuntamente la volontà di scioglimento dinanzi all'ufficiale dello stato civile. In tale caso la domanda di scioglimento dell'unione civile è proposta decorsi tre mesi dalla data della manifestazione di volontà di scioglimento dell'unione». Disciplina che, pur ricalcando quanto previsto per il matrimonio dal Código Civil spagnolo e nell'ottica di una semplificazione dello scioglimento del vincolo (matrimonio ovvero unione civile) che non interessa solo l'Italia, bensì l'Europa, presenta comunque tratti di censurabilità costituzionale. Se è pur vero che il legislatore ha recentemente abbreviato i termini per giungere al matrimonio, abbreviando i termini della separazione (consensuale ovvero giudiziale), è pur vero che rimane, ad oggi, incomprensibile la disparità di trattamento, sia con riferimento a quanto previsto all'art.1 c. 24 , 1. n. $76 / 2016$, di cui sopra (disciplina assente per l'istituto del matrimonio in Italia), sia con riferimento ai differenti termini previsti tra manifestazione della volontà di separazione e domanda di scioglimento del vincolo giuridico (3 mesi per le unioni civili, 6 o 12

47 Sulla risarcibilità dei danni ex art. 2043 c.c. per violazione dell'obbligo di fedeltà di cui all'art. 143, c. 2, c.c. si cfr., ex multis, Cass. 18853/2011; si potrebbe, inoltre, ipotizzare che la giurisprudenza inauguri un'interpretazione dell'art. 1 c. 11, 1. n. 76/2016, che, se la fattispecie lo consenta, riconduca il tradimento alla violazione dell'obbligo di assistenza morale. Sull'obbligo di assistenza morale e ciò che ad esso possa essere ricondotto si veda: P. Zatti, «I diritti e i doveri che nascono dal matrimonio e la separazione dei coniugi, in Tratt. Rescigno, UTET, Torino, 1982, p. 45. 
mesi per il matrimonio a seconda, rispettivamente, che la separazione sia consensuale ovvero giudiziale $)^{48}$.

\section{LA DISCIPLINA DELL'ADOZIONE PER LE COPPIE SAME SEX: SPAGNA VS. ITALIA}

E' possibile, ad oggi, affermare che la disciplina nei due Stati sia agli antipodi. Se, infatti, in Spagna, con la sentenza del 2012, il Tribunal Constitucional ha ribadito la legittimità costituzionale della normativa che estende l'applicazione della disciplina dell'adozione congiunta anche alle coppie omosessuali, in Italia detta disciplina (nella sola declinazione della Stepchild Adoption) è stata stralciata dal disegno di legge, al fine della sua stessa approvazione.

Con sentenza del 6 novembre n. 198 del 2012 il TC ha, infatti, stabilito che la nuova redazione dell'art. 117, c. 4, del Código Civil, è conforme a quanto previsto all'art. $39^{49}$, della Constitución Española: dato che l'ordinamento spagnolo prevede che il giudice, nell'emettere sentenza, ha il dovere di valutare l'interesse

${ }^{48}$ Ai sensi dell'art. 81 del Código Civil, i termini per richiedere la separazione in Spagna sono i medesimi previsti in Italia per lo scioglimento dell'unione civile; l'articolo comprende inoltre la medesima possibilità disciplinata dall'art. 1, c. 241 . n. 76/2016 con riferimento alla possibilità di espressione di volontà disgiunta soggetta ai medesimi termini: «Se decretará judicialmente la separación cuando existan hijos menores no emancipados o con la capacidad modificada judicialmente que dependan de sus progenitores, cualquiera que sea la forma de celebración del matrimonio: $1 .^{\circ}$ A petición de ambos cónyuges o de uno con el consentimiento del otro, una vez transcurridos tres meses desde la celebración del matrimonio. A la demanda se acompañará una propuesta de convenio regulador redactada conforme al artículo 90 de este Código. 2. ${ }^{\circ}$ A petición de uno solo de los cónyuges, una vez transcurridos tres meses desde la celebración del matrimonio. No será preciso el transcurso de este plazo para la interposición de la demanda cuando se acredite la existencia de un riesgo para la vida, la integridad física, la libertad, la integridad moral o libertad e indemnidad sexual del cónyuge demandante o de los hijos de ambos o de cualquiera de los miembros del matrimonio. A la demanda se acompañará propuesta fundada de las medidas que hayan de regular los efectos derivados de la separación». Oggi, in Spagna, successivamente alla riforma del 2005, non è più necessario un periodo di separazione per chiedere il divorzio, né che lo stesso sia motivato. E' sufficiente che al momento della richiesta, anche unilaterale, siano passati 3 mesi dal momento della celebrazione del matrimonio. Pertanto l'art. 81, oggi, viene richiamato dall'art. 86, meramente per stabilire circostanze e requisiti in caso di separazione (come già sottolineato, non più necessaria, preordinata nè prodromica alla richiesta di divorzio). Il Codigo Civil così stabilisce: - art.86: «Se decretará judicialmente el divorcio, cualquiera que sea la forma de celebración del matrimonio, a petición de uno solo de los cónyuges, de ambos o de uno con el consentimiento del otro, cuando concurran los requisitos y circunstancias exigidos en el artículo 81»; - Altresì, art.85: «El matrimonio se disuelve, sea cual fuere la forma y el tiempo de su celebración, por la muerte o la declaración de fallecimiento de uno de los cónyuges y por el divorcio».

${ }^{49}$ Lart. 39 della Constitución española così recita: «1. Los poderes públicos aseguran la protección social, económica y jurídica de la familia. 2. Los poderes públicos aseguran, asimismo, la protección integral de los hijos, iguales éstos ante la ley con independencia de su filiación, y de las madres, cualquiera que sea su estado civil. La ley posibilitará la investigación de la paternidad. 3. Los padres deben prestar asistencia de todo orden a los hijos habidos dentro o fuera del matrimonio, 
dell'adottato e l'idoneità dell'adottante/adottanti ${ }^{50}$, il TC considera che l'interesse del minore venga preservato, sia che lo stesso sia adottato da una famiglia di orientamento eterosessuale ovvero omosessuale, specificando, per così dire "a garanzia» della propria posizione e a «rassicurazione» delle posizioni contrarie alla decisione medesima -plurimamente-che l'interesse ultimo rimane sempre quello del minore. Il TC specifica, inoltre, come l'adozione a titolo individuale fosse già contemplata dall'ordinamento spagnolo, e tra i requisiti per la valutazione dell'idoneità dell'adottante, non sussista l'aver contratto matrimonio ovvero l'orientamento sessuale del medesimo.

In Italia, al contrario, agli uniti civilmente non è consentito accedere, ex art. 1, c. 20 (primo e secondo periodo), ${ }^{51}$ legge n. 76/2016 all'istituto dell'adozione ex l. n. 184/83 (e successive modifiche) -fatto salvo, ai sensi dell'art. 1, c. 20, ultimo periodo ${ }^{52}$, quanto previsto per i casi di adozioni in casi particolari di cui all'art. 44 della legge n. $184 / 83^{53}$.

durante su minoría de edad y en los demás casos en que legalmente proceda. 4. Los niños gozarán de la protección prevista en los acuerdos internacionales que velan por sus derechos.

${ }^{50}$ Il TC specifica, ad ogni modo, che non esiste, ad oggi, un diritto fondamentale ad adottare, bensì un diritto ad essere adottati, citando, altresì, la sentenza della Corte EDU «Frettè c. Francia», ricorso n. 36515/97, sentenza del 26 febbraio 2002: «L'adozione è dare una famiglia a d un figlio, non un figlio ad una famiglia. Lo Stato deve assicurarsi che le persone scelte come adottanti siano quelle che possono offrire, da tutti i punti di vista, le condizioni di accoglienza più favorevoli»; e ancora "Le autorità nazionali [...] dispongono di un ampio margine quando sono chiamati a pronunciarsi in questo ambito [...] le autorità nazionali sono, in principio, in una posizione migliore rispetto a quella di una giurisdizione internazionale per valutare le sensibilità e i contesti locali». Si evidenzia inoltre come altra sentenza della Corte Edu -E.B. c. Francia, ricorso n. 43546/02, sentenza del 22 gennaio 2008- sancisca come il diniego di concedere l'autorizzazione all'adozione di un bambino, in ragione dell'orientamento sessuale dell'adottante, costituisca violazione dell'art. 14 CEDU (letto in combinato disposto con l'art. $8 \mathrm{CEDU}$ ), in un sistema normativo -nel caso di specie quello francese- che consente l'adozione da parte dei single.

${ }^{51}$ Art. 1, c. 20,1. n. 76/2016, primo e secondo periodo: «Al solo fine di assicurare l'effettività della tutela dei diritti e il pieno adempimento degli obblighi derivanti dall'unione civile tra persone dello stesso sesso, le disposizioni che si riferiscono al matrimonio e le disposizioni contenenti le parole "coniuge», "coniugi» o termini equivalenti, ovunque ricorrono nelle leggi, negli atti aventi forza di legge, nei regolamenti nonché negli atti amministrativi e nei contratti collettivi, si applicano anche ad ognuna delle parti dell'unione civile tra persone dello stesso sesso. La disposizione di cui al periodo precedente non si applica alle norme del codice civile non richiamate espressamente nella presente legge, nonché alle disposizioni di cui alla legge 4 maggio 1983, n. 184». Si segnala, inoltre, la sentenza CtEDU (Grande Camera della Corte Europea per i diritti dell'uomo) del 19 febbraio 2013, X e altri c. Austria, che dichiara non conforme a CEDU la previsione contenuta nella legislazione nazionale austriaca che consentiva l'adozione alle coppie eterosessuali, anche se non coniugate, ma non già a coppie omosessuali non coniugate, ravvisando in detta previsione legislazione una violazione del combinato disposto degli art.. 8 e 14 della Convenzione, rilevando così una discriminazione a causa dell'orientamento sessuale dell'adottante.

52 Art.1 c.20, l.n.76/2016, ultimo periodo: «Resta fermo quanto previsto e consentito in materia di adozione dalle norme vigenti».

53 Tale espresso rinvio legale -secondo l'interpretazione corrente- sembrerebbe, ad ogni modo, avallare la tesi secondo cui la lettura dell'art. 44, lett. d), della legge n. 184/83 prevalente in 


\section{CONCLUSIONI}

Dall'analisi sopra esplicitata si evidenzia quindi come la disciplina legislativa nazionale italiana, pur se a tratti passibile di censura costituzionale, è conforme alla normativa europea e alla giurisprudenza della Corte Edu, pur nella consapevolezza che essa riconosce pressoché solamente i minimi livelli di tutela previsti a livello internazionale.

La Spagna, di contro, pur essendo lo stesso Paese dell'area mediterranea con radici storico-giuridico-culturali affini a quelle italiane, è, nella tematica in esame, agli antipodi.

Con la ley n. 13/2005, riconosce (tra i primi Paesi in Europa a farlo) il massimo livello di tutela alle coppie omosessuali, equiparando le stesse e da un punto di vista formale, e da un punto di vista sostanziale, alle coppie eterosessuali, e, più in generale disponendo di una disciplina del diritto privato di famiglia caratterizzata da una visione moderna dello stesso, conforme e coerente con l'evoluzione sociale del concetto di famiglia e dei nuclei in cui la stessa si forma e si sviluppa.

Ad ogni modo, in conclusione, pur nel libero apprezzamento comunitariamente concesso e previsto per ogni legislazione nazionale, ciò che rileva sottolineare è l'uniformarsi e il convergere dei Paesi di matrice europea nel riconoscimento dei diritti fondamentali per lo sviluppo del sé nelle formazioni sociali, unitamente al concreto tentativo (non -ancora- ovunque compiuto) di eliminare dall'ordinamento (e dalla società?) le discriminazioni concernenti l'orientamento e le scelte riguardanti la sfera sessuale, impeditive di un pieno sviluppo dell'individuo in ogni ambito della propria personalità.

ReCibido: diciembre de 2017, ACEPTADo: abril de 2018

giurisprudenza debba essere mantenuta pur al netto dello stralcio dalla Legge Cirinnà della «Stepchild Adoption». Infatti, la Corte di cassazione n. 12962/2012 ha ritenuto ammissibile l'adozione del figlio del partner non già in base alle lett. b) dell'art. 44 , ma alla luce di un'interpretazione della lett. d) dell'art. 44 della legge n. 184/83, secondo la quale: «la nozione di «impossibilità di affidamento preadottivo", va intesa non solo come impossibilità «di fatto», ma anche come impossibilità «di diritto»-intendendosi tale quella derivante dalla mancanza dei presupposti giuridici per procedere all'adozione "piena» - e sempre che risulti accertato in fatto- secondo il disposto dell'art. 57, l. adoz. -che il ricorso all'adozione, sia pur nella sua forma «minor», corrisponda al preminente interesse del bambino, in quanto formalizzazione di una relazione affettiva già esistente e valutata nel corso dell'istruttoria come elemento positivo nella sua crescita [...]». 